\title{
Will an Aspirin a Day Keep Cancer Away?
}

Data suggesting that regular aspirin use lowers cancer risk has accumulated to the point where some argue that it's time to recommend that many more people take the drug

IN THE LATE 1970S, A SURGEON IN

Melbourne, Australia, wanted to figure out why his country had a relatively high rate of colorectal cancer. After he and colleagues interviewed more than 700 cancer patients and a comparable number of healthy people, they reported in 1987 and 1988 that Australians' penchant for beer, fatty foods, and red meat all seemed to predispose them to the disease. The researchers also found a surprising protective factor: People who regularly used aspirin were $40 \%$ less likely to develop colorectal cancer than those who didn't take the drug.

That first hint that the age-old headache remedy also blocks intestinal tumors helped spur a wave of research in animals and clinical trials that established that aspirin and other nonsteroidal anti-inflammatory drugs (NSAIDs) protect against colon cancer. And now, 2 decades later, aspirin is generating new excitement among cancer researchers. A series of studies from the United Kingdom in the last 2 years has offered the first evidence from placebo-controlled clinical trials that regularly taking low doses of aspirin wards off other types of cancer as well.

The studies, which tallied cancer cases among people who had been taking aspirin for years to prevent vascular events such as heart attack and stroke, found that death rates from several tumor types were as much as $37 \%$ lower. And even in the people who developed a cancer, taking aspirin seemed to slow the spread of tumors to other parts of the body. "It's just about the first proof of principle that a simple compound of any kind can reduce the risk of several cancers," says lead researcher Peter Rothwell of the University of Oxford in the United Kingdom.

These reports have raised the tantalizing possibility that aspirin could serve as the first anticancer drug for the general population. "It reshapes the debate about the risks and benefits of aspirin for cancer prevention," says colorectal cancer researcher Andrew Chan of Massachusetts General Hospital in Boston.
Because aspirin can cause stomach upset and dangerous internal bleeding, U.S. guidelines now recommend that only people at elevated risk for heart disease or stroke take low doses of the medicine, typically 81 milligrams a day. But Chan and others suggest that medical societies and policymakers should also consider aspirin's general cancer-fighting other NSAIDs protect against cancer (see sidebar, p. 1472). NCI hopes the attempt to resolve the question - there are several competing theories - will lead to next-generation anticancer drugs and biomarkers showing who will respond to them.

But some cancer researchers say health policymakers needn't wait for a better aspirin and should advocate wider use of the current one. "This is a really extraordinary opportunity if everything bears out, because you have a drug that costs a penny a pill at a low dose, that can prevent the two major causes of death in the Western world," says epidemiologist Michael Thun of the American Cancer Society in Atlanta.

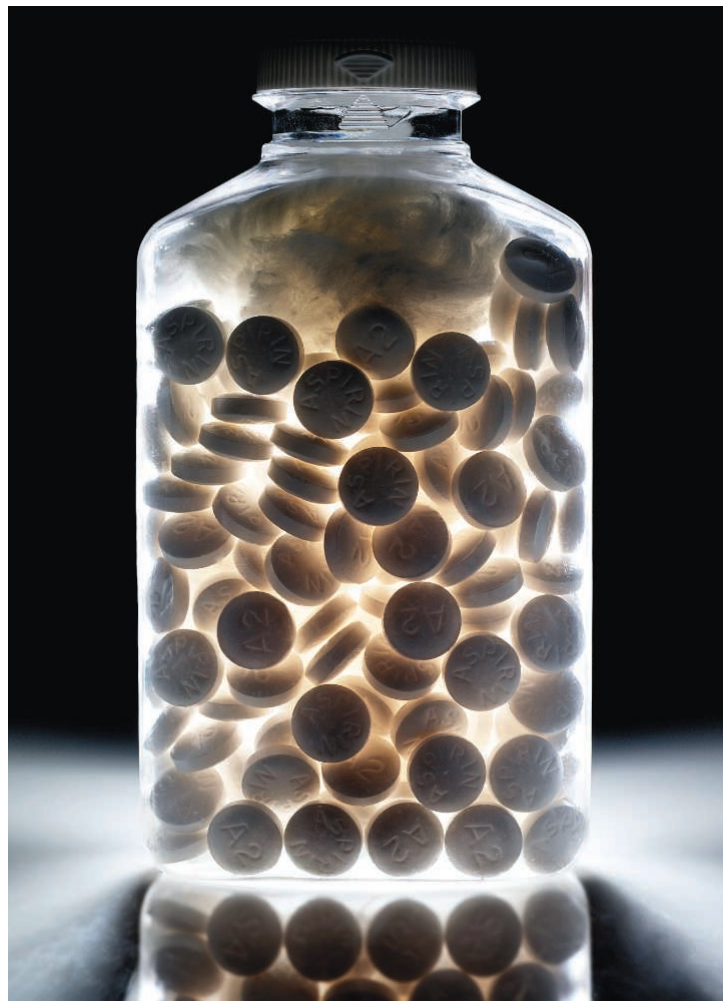

Versatile. Aspirin may block cancer as well as vascular disease.

\section{Comeback}

Thun's optimism is the latest high in the up-and-down story of NSAIDs as a cancer preventive. Aspirin and some other NSAIDs first bore out their promise in trials published starting in 2000 among people who had had precancerous colon polyps removed and others genetically prone to colorectal cancer. The drugs protected them from polyps and premalignant tumors that precede full-blown cancer. Such people are now sometimes advised by their doctors to take NSAIDs as an adjunct to surgery to prevent polyps from recurring.

Although epidemiological evidence has suggested that aspirin could have broader anticancer effects, those results aren't conclusive. They come from studies in which people answered questions about their past use of medicationsa design prone to bias in part because memories aren't reliable. And hopes for aspirin fell in 2005 when a huge prospective study - a randomized controlled trial called the Women's Health Study (WHS) - failed to show a reduction in the incidence of cancer in nearly effects. Rothwell, who is 48 , is so convinced by his team's data, for example, that he's begun taking aspirin daily even though he has no risk factors for vascular disease.

The new British aspirin studies are also fueling basic research on NSAIDs to ward off cancer, a field that lost momentum in the past decade when one NSAID, Vioxx, was pulled off the market because of safety concerns. Partly because of the U.K. results, U.S. National Cancer Institute (NCI) Director Harold Varmus last year added to his list of 24 "Provocative Questions" one that asks what is the mechanism by which aspirin and 40,000 women who took low-dose aspirin every other day for 10 years.

The field of NSAIDs for cancer prevention also suffered a black eye when two arthritis drugs developed to avoid the side effects of aspirin, the COX-2 inhibitors Vioxx and Celebrex, were tested in trials to prevent colon polyps. In one large trial, Vioxx cut the risk of polyps but raised the chances of heart attack so much that risk arguably outweighed the cancer benefit. Vioxx was pulled off the market in 2004 and Celebrex, although still used for preventing colorectal cancer in people at high risk of such disease, now carries a 
DISEASE PREVENTION है www.sciencemag.org/special/prevention

"black box" warning label.

The pendulum began to swing back in favor of aspirin, however, when Rothwell, a neurologist, noticed that fewer overall cancers had occurred among patients in the treatment arm of randomized trials launched in the 1980s to test aspirin as a way to prevent stroke. Amassing details on the cancers from patients' paper health records and U.K. health databases, his team initially confirmed aspirin's ability to ward off colorectal cancers, reporting in 2007 that there were $24 \%$ fewer cases and 35\% fewer deaths in the aspirin group after several years. Then in late 2010, they reported that patients taking aspirin at any dose daily for at least 5 years cut by $21 \%$ the long-term risk of dying from colorectal, gastrointestinal, esophageal, stomach, pancreatic, brain, lung, and prostate cancers.

Rothwell's group expanded on these results in three papers published in The Lancet and The Lancet Oncology this spring. One, a meta-analysis of 51 randomized trials in which aspirin was taken daily, found $37 \%$ fewer deaths from cancers after
Believer. Peter Rothwell's cancer studies convinced him to take a daily aspirin.

5 years. (The study came with a large caveat: It excluded WHS and an earlier trial that found no benefit, the Physicians' Health Study, because participants in each took aspirin only every other day.) This meta-analysis also found that while the aspirin group had more stomach bleeds, these incidents were not fatal-people recovered - and the bleeding risk went down after several years on aspirin. Another paper found that people on aspirin who developed cancer were $36 \%$ less likely to have tumors that had spread. A third paper found a "remarkable consistency" in the drop in cancers among aspirin users in epidemiologic studies and clinical trials, Rothwell says.

While these studies weren't designed specifically to test aspirin against cancer, some researchers say that because so many people now take aspirin off-label it would be diffi-

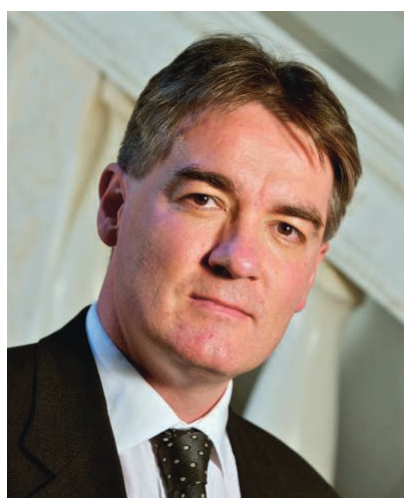

cult to conduct better trials. The Rothwell studies are "probably the best evidence we'll ever have on this topic," Chan says.

\section{Weighing risks}

Chan is part of an international panel on cancer prevention that, in response to the Rothwell studies, plans to update its stance on aspirin published 3 years ago. The group's leader, epidemiologist Jack Cuzick of Queen Mary, University of London expects the panel may suggest that people take low doses of aspirin daily starting around age 50 and stopping by age 70 , when the risk of internal bleeding rises. The group may also discuss whether doctors should screen patients for the ulcer-causing Helicobacter pylori bacterium, treating those who test positive with antibiotics before putting them on aspirin, to reduce the risk of bleeds.

Some U.S. researchers also suggest that it's time for the U.S. Preventive Services

\section{Wondering How the Wonder Drug Works}

HOW DOES TAKING ASPIRIN WARD OFF CANCER? RESEARCHERS still don't understand the mechanism, a lack of knowledge that threatens to frustrate development of better cancer prevention agents. But some say the latest clinical studies linking low-dose aspirin use with less cancer (see main story, p. 1471) suggest there's no need to improve upon this 113-year-old workhorse drug.

Among its many known effects, aspirin, or acetylsalicylic acid, inhibits two forms of enzymes known as cyclooxygenases (COX) that convert arachidonic acid into lipids called prostaglandins. Prostaglandins made by COX-1 protect the stomach lining, while those made

by COX-2 are involved in inflammation. Aspirin's inhibitor effects on COX-1 seem to explain why the medicine can upset stomachs and trigger internal bleeding. Many researchers have concluded that aspirin prevents cancer mainly by blocking the activity of COX-2. That's because the same inflammation-driven responses that help tissue recovery from wound injury-cell division, blood vessel formation, and suppression of programmed cell death, for example-may also help tumors grow.

Several lines of evidence implicate COX-2 in cancer induction. The enzyme is overproduced in many cancer types; mice lacking its gene are less prone to colon cancer; and trials of nonsteroidal anti-inflammatory drugs (NSAIDs) that target only COX-2 protected against precancerous colon polyps in people at high risk.

Reducing inflammation via COX-2 probably isn't the only way aspirin prevents cancer, however. "We are as a field getting down to some of the mechanisms, but it's very complicated," says Raymond Dubois of the MD Anderson Cancer Center in Houston, Texas. A decade ago, researchers found that aspirin can block production of a protein called
NF- $\kappa B$ that protects cells from early death. Although the studies were done in the test tube at aspirin doses much higher than those in the bloodstream, the NF- $\kappa$ B hypothesis remains on the table-along with more than a dozen possible mechanisms-from inhibiting various cell growth pathways to targeting cancer stem cells. "There are a number of very different, divergent ideas," says Asad Umar of the National Cancer Institute's Division of Cancer Prevention in Bethesda, Maryland.

Some experts say new clinical studies of low-dose aspirin and cancer suggest that COX-2 isn't directly involved at all. That's because at low doses, the drug doesn't block COX-2 but still impairs platelets, the small cell fragments in blood that help form clots, via the COX-1 pathway. Studies suggest that platelets blunt the immune attack on cancer cells traveling in blood and produce growth factors that help them take root in a new place, notes University of Pennsylvania pharmacologist Garrett FitzGerald. Some experiments suggest that activated platelets can also stimulate the COX-2 pathway in adjacent cells, which would explain how aspirin, by damping COX-2 at the site of an injury, could block the early stages of colorectal cancer, says Carlo Patrono, a pharmacologist at the Catholic University in Rome, Italy.

Efforts to make an alternative to standard aspirin haven't yet panned out. Several drugs that target only COX-2, notably Vioxx and Celebrex, unacceptably raised heart attack risk, for example. And a version of acetylsalicylic acid containing a chemical group called nitric oxide that was supposed to reduce side effects failed to gain regulatory approval. Some researchers are combining aspirin with other compounds such as a lipid to reduce stomach upset. But these are years from the clinic. For now, "I don't think a better aspirin is the issue," but rather figuring out which individuals stand to benefit from it most, FitzGerald says. -J.K. 
Task Force (see p. 1468) to update its guidelines on the risks and benefits of daily aspirin use. The group had endorsed its preventive prowess for heart attack and stroke but discounted its anticancer effects. The potential to protect against both cancer and heart disease could tip the balance toward recommending aspirin for many more healthy adults, Thun says.

Others are more cautious about recommending broad use of aspirin to ward off can-
"It reshapes the debate about the risk and benefits of aspirin for cancer prevention,"

-ANDREW CHAN, MASSACHUSETTS GENERAL HOSPITAL cer. "In 2012, gastroenterologists still get called to the emergency room for bleeds" caused by the drug, says cancer prevention researcher Andrew Dannenberg of Cornell University. Colorectal cancer researcher Sanford Markowitz of Case Western Reserve University in Cleveland, Ohio, points to studies suggesting that only people with a particular genetic profile will see their cancer risk go down if they take aspirin. "I would want more data on who benefits and who does not," Markowitz says.

Cancer prevention researcher Raymond Dubois of the MD Anderson Cancer Center in Houston, Texas, is also wary. "Initially we thought we could put aspirin in the drinking water. That's not the case," he says. "Where this field is going is towards a more personalized approach."

The picture may become clearer soon when WHS reports on longer-term effects of aspirin on cancer risk. Their next paper "will be crucial," Rothwell says. And even some on the pro-aspirin side urge caution. "We don't want to mess this up," Thun says.

-JOCELYN KAISER
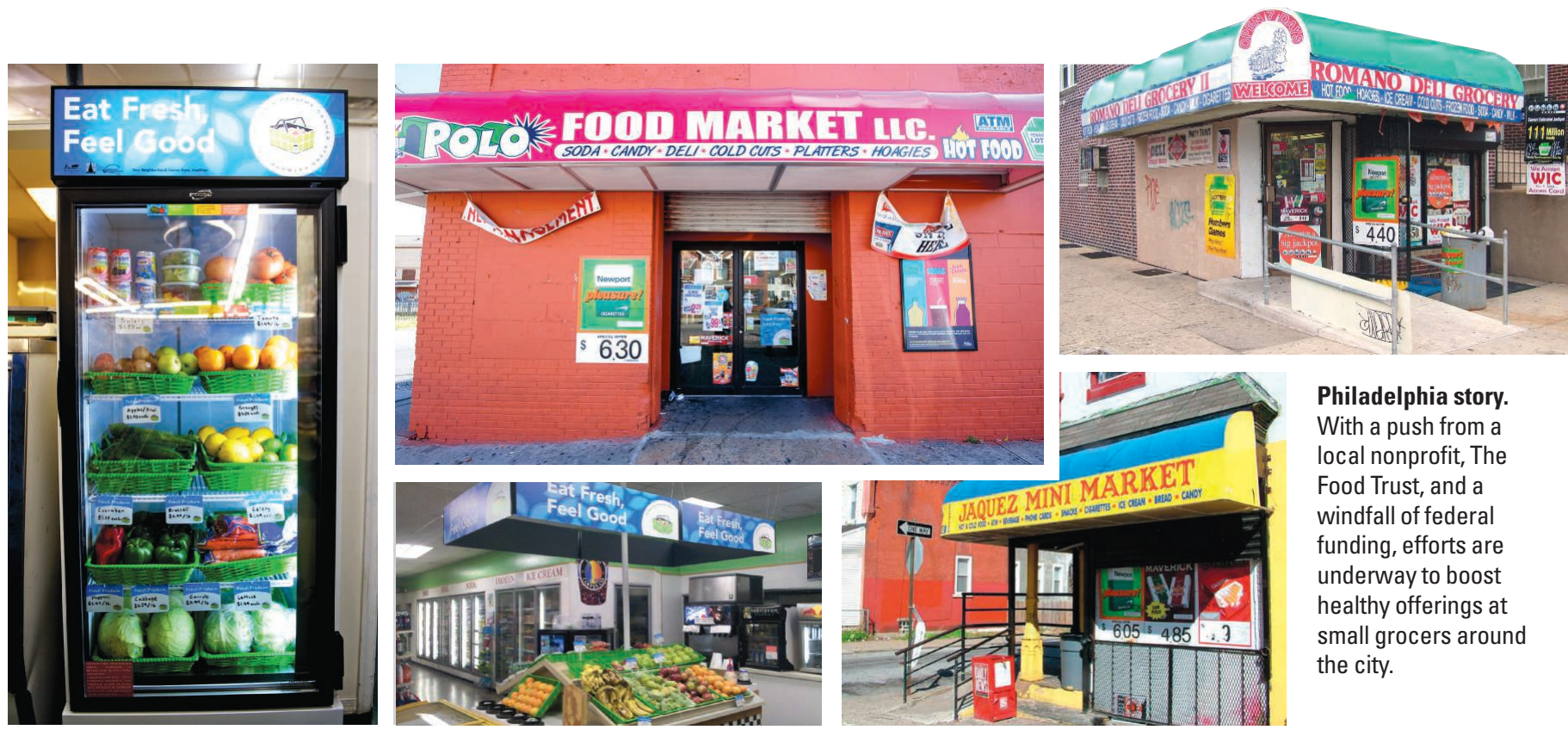

Philadelphia story.

With a push from a local nonprofit, The Food Trust, and a windfall of federal funding, efforts are underway to boost healthy offerings at small grocers around the city.

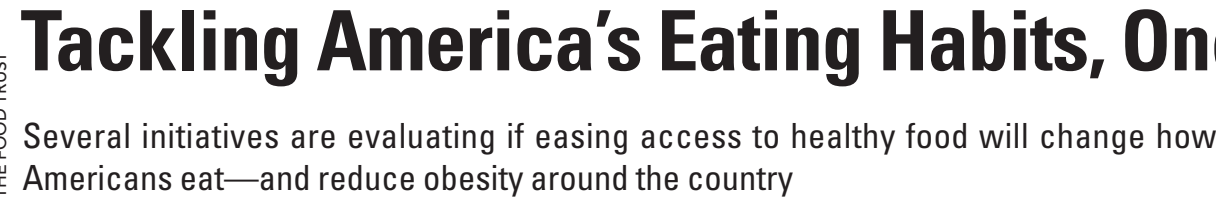

PHILADELPHIA, PENNSYLVANIA-The food market Clara Santos runs in South Philadelphia is less than 5 kilometers from the city's majestic art museum. Driving there, polished brick townhouses and trendy eateries with names like Caffeination and The Belgian Café give way to stretches of modest row houses, abandoned lots, and the occasional corner store advertising massive sandwiches called hoagies. Santos, who emigrated from the Dominican Republic, lives not far from the Olivares Food Market she took over 4 years ago. Graffiti marks its outer walls, but the shop, on a quiet block sandwiched between two city schools, is tidy inside. Children stop in frequently for soda and candy after classes let out.

Santos's store is one of 642 data points in an unusual urban experiment. Eight years ago, a local nonprofit called The Food Trust began studying whether bringing healthy, affordable food to corner stores like hers could change eating habits among the city's economically disadvantaged residents. The program started small. Then in 2010, the Healthy Corner Store Initiative rapidly expanded after partnering with the Department of Public Health here, which had just received a windfall:
$\$ 15$ million to help prevent obesity, part of $\$ 373$ million from the federal stimulus package earmarked for health and wellness efforts around the country. Santos joined the project last year along with hundreds of other convenience store owners. Among other support, she received a display refrigerator where she showcases yogurt, precut watermelon, carrots, apples, and other fruits and vegetables, along with green bins for produce such as onions and plantains. Why not give the program a try, Santos says she thought: "Maybe we'll help the community."

Like Santos and those at The Food Trust, more and more people are trying to change the landscape of food in America. And change is desperately needed. More than one-third of 